\title{
Formulation of Electrodynamics with an External Source in the Presence of a Minimal Measurable Length
}

\author{
S. K. Moayedi, ${ }^{1}$ M. R. Setare, ${ }^{2}$ and B. Khosropour ${ }^{1}$ \\ ${ }^{1}$ Department of Physics, Faculty of Sciences, Arak University, Arak 38156-8-8349, Iran \\ ${ }^{2}$ Department of Science, Campus of Bijar, University of Kurdistan, Bijar, Iran
}

Correspondence should be addressed to M. R. Setare; rezakord@ipm.ir

Received 13 December 2012; Accepted 18 February 2013

Academic Editor: Frederik G. Scholtz

Copyright (c) 2013 S. K. Moayedi et al. This is an open access article distributed under the Creative Commons Attribution License, which permits unrestricted use, distribution, and reproduction in any medium, provided the original work is properly cited.

\begin{abstract}
In a series of papers, Quesne and Tkachuk (2006) presented a D + 1-dimensional $\left(\beta, \beta^{\prime}\right)$-two-parameter Lorentz-covariant deformed algebra which leads to a nonzero minimal measurable length. In this paper, the Lagrangian formulation of electrodynamics in a $3+$ 1-dimensional spacetime described by Quesne-Tkachuk algebra is studied in the special case of $\beta^{\prime}=2 \beta$ up to the first order over the deformation parameter $\beta$. It is demonstrated that at the classical level there is a similarity between electrodynamics in the presence of a minimal measurable length (generalized electrodynamics) and Lee-Wick electrodynamics. We obtain the free space solutions of the inhomogeneous Maxwell's equations in the presence of a minimal length. These solutions describe two vector particles (a massless vector particle and a massive vector particle). We estimate two different upper bounds on the isotropic minimal length. The first upper bound is near to the electroweak length scale $\left(\ell_{\text {electroweak }} \sim 10^{-18} \mathrm{~m}\right)$, while the second one is near to the length scale for the strong interactions $\left(\ell_{\text {strong }} \sim 10^{-15} \mathrm{~m}\right)$. The relationship between the Gaete-Spallucci nonlocal electrodynamics $(2012)$ and electrodynamics with a minimal length is investigated.
\end{abstract}

\section{Introduction}

The unification between the general theory of relativity and the standard model of particle physics is one of the most important problems in theoretical physics [1]. This unification predicts the existence of a minimal measurable length on the order of the Planck length. Also, recent studies in perturbative string theory and loop quantum gravity suggest that there is a minimal length scale in nature [2].

Today we know that the existence of a minimal measurable length leads to an extended uncertainty principle. This extended uncertainty principle can be written as

$$
\Delta X \Delta P \geq \frac{\hbar}{2}\left[1+a_{1}\left(\frac{l_{P}}{\hbar}\right)^{2}(\Delta P)^{2}+a_{2}\left(\frac{l_{P}}{\hbar}\right)^{4}(\Delta P)^{4}+\cdots\right],
$$

where $l_{P}$ is the Planck length and $a_{i}, \forall i \in\{1,2, \ldots\}$, are positive numerical constants $[3,4]$. If we keep only the first two terms on the right-hand side of (1), we will obtain the usual generalized uncertainty principle (GUP) as follows:

$$
\Delta X \Delta P \geq \frac{\hbar}{2}\left[1+a_{1}\left(\frac{l_{P}}{\hbar}\right)^{2}(\Delta P)^{2}\right] .
$$

It is obvious that in (2), $\Delta X$ is always greater than $(\Delta X)_{\min }=$ $\sqrt{a_{1}} l_{P}$. Many physicists believe that reformulation of quantum field theory in the presence of a minimal measurable length leads to a divergence free quantum field theory [5-7]. In the recent years, reformulations of quantum mechanics, gravity, and quantum field theory in the presence of a minimal measurable length have been studied extensively [4-18]. The first attempt to construct the electromagnetic field in quantized spacetime was made by Snyder [19]. In a previous work [14], we studied formulation of an electrostatic field with a charge density in the presence of a minimal length based on the Kempf algebra. In the present work, we study formulation of electrodynamics with an external source in the presence of a minimal measurable length based on the Quesne-Tkachuk algebra. The organization of our 
paper is as follows. In Section 2, the $D+1$-dimensional $\left(\beta, \beta^{\prime}\right)$-two-parameter Lorentz-covariant deformed algebra introduced by Quesne and Tkachuk is studied and it is shown that the Quesne-Tkachuk algebra leads to a minimal measurable length $[20,21]$. In Section 3, the Lagrangian formulation of electrodynamics with an external source in a $3+1$-dimensional spacetime described by Quesne-Tkachuk algebra is introduced in the special case of $\beta^{\prime}=2 \beta$, in which the position operators commute to the first order in $\beta$. We show that at the classical level there is a similarity between electrodynamics in the presence of a minimal measurable length and Lee-Wick electrodynamics. In Section 4, the free space solutions of the inhomogeneous Maxwell's equations in the presence of a minimal measurable length are obtained. These solutions describe two different particles, a massless vector particle and a massive vector particle. In Section 5, we obtain two different upper bounds on the isotropic minimal length. One of these upper bounds on the isotropic minimal length is near to the electroweak length scale $\left(\ell_{\text {electroweak }} \sim\right.$ $\left.10^{-18} \mathrm{~m}\right)$. The second upper bound on the isotropic minimal length is near to the length scale for the strong interactions $\left(\ell_{\text {strong }} \sim 10^{-15} \mathrm{~m}\right)$. In Section 6 , we investigate the relation between electrodynamics in the presence of a minimal measurable length and the concept of nonlocality in electrodynamics. Our conclusions are presented in Section 7. SI units are used throughout this paper.

\section{Lorentz-Covariant Deformed Algebra with a Minimal Observable Distance}

Recently, Quesne and Tkachuk have introduced a Lorentzcovariant deformed algebra which describes a $D+1$ dimensional quantized spacetime [20, 21]. The QuesneTkachuk algebra in a $D+1$-dimensional spacetime is specified by the following generalized commutation relations:

$$
\begin{gathered}
{\left[X^{\mu}, P^{\nu}\right]=-i \hbar\left[\left(1-\beta P_{\rho} P^{\rho}\right) g^{\mu \nu}-\beta^{\prime} P^{\mu} P^{\nu}\right]} \\
{\left[X^{\mu}, X^{\nu}\right]=i \hbar \frac{2 \beta-\beta^{\prime}-\left(2 \beta+\beta^{\prime}\right) \beta P_{\rho} P^{\rho}}{1-\beta P_{\rho} P^{\rho}}\left(P^{\mu} X^{\nu}-P^{\nu} X^{\mu}\right)} \\
{\left[P^{\mu}, P^{v}\right]=0,}
\end{gathered}
$$

where $\mu, v, \rho=0,1,2, \ldots, D$ and $\beta$ and $\beta^{\prime}$ are two nonnegative deformation parameters $\left(\beta, \beta^{\prime} \geq 0\right)$. In the above equations, $\beta$ and $\beta^{\prime}$ are constant parameters with dimension (momentum) $)^{-2}$. Also, $X^{\mu}$ and $P^{\mu}$ are position and momentum operators in the deformed space and $g_{\mu \nu}=g^{\mu \nu}=$ $\operatorname{diag}(1,-1,-1, \ldots,-1)$. In the special case where $D=3$ and $\beta=0$, the Quesne-Tkachuk algebra (3)-(5) reduces to the Snyder algebra [22].

An immediate consequence of relation (3) is the appearance of an isotropic minimal length which is given by

$$
\left(\Delta X^{i}\right)_{0}=\hbar \sqrt{\left(D \beta+\beta^{\prime}\right)\left[1-\beta\left\langle\left(P^{0}\right)^{2}\right\rangle\right]}, \quad \forall i \in\{1,2, \ldots, D\} \text {. }
$$

In [23], Tkachuk introduced a representation which satisfies the generalized commutation relations (3)-(5) up to the first order in deformation parameters $\beta$ and $\beta^{\prime}$.

The Tkachuk representation is given by

$$
\begin{gathered}
X^{\mu}=x^{\mu}-\frac{2 \beta-\beta^{\prime}}{4}\left(x^{\mu} p_{\rho} p^{\rho}+p_{\rho} p^{\rho} x^{\mu}\right), \\
P^{\mu}=\left(1-\frac{\beta^{\prime}}{2} p_{\rho} p^{\rho}\right) p^{\mu}
\end{gathered}
$$

where $x^{\mu}$ and $p^{\mu}=i \hbar\left(\partial / \partial x_{\mu}\right)=i \hbar \partial^{\mu}$ are position and momentum operators in ordinary relativistic quantum mechanics. In this study, we consider the special case of $\beta^{\prime}=$ $2 \beta$, in which the position operators commute to the first order in deformation parameter $\beta$, that is, $\left[X^{\mu}, X^{\nu}\right]=0$. In this linear approximation, the Quesne-Tkachuk algebra becomes

$$
\begin{gathered}
{\left[X^{\mu}, P^{\nu}\right]=-i \hbar\left[\left(1-\beta P_{\rho} P^{\rho}\right) g^{\mu \nu}-2 \beta P^{\mu} P^{\nu}\right],} \\
{\left[X^{\mu}, X^{\nu}\right]=0,} \\
{\left[P^{\mu}, P^{\nu}\right]=0 .}
\end{gathered}
$$

The following representations satisfy (8), in the first order in $\beta$ :

$$
\begin{gathered}
X^{\mu}=x^{\mu}, \\
P^{\mu}=\left(1-\beta p_{\rho} p^{\rho}\right) p^{\mu} .
\end{gathered}
$$

Note that the representations (7) and (9) coincide when $\beta^{\prime}=$ $2 \beta$.

\section{Lagrangian Formulation of Electrodynamics with an External Source in the Presence of a Minimal Length Based on the Quesne-Tkachuk Algebra}

The Lagrangian density for a massless vector field $A^{\mu}=$ $((1 / c) \phi, \mathbf{A})$ with an external source $J^{\mu}=(c \rho, \mathbf{J})$ in a $3+1$ dimensional spacetime is [24]

$$
\mathscr{L}=-\frac{1}{4 \mu_{0}} F_{\mu \nu} F^{\mu \nu}-J^{\mu} A_{\mu},
$$

where $F_{\mu \nu}=\partial_{\mu} A_{\nu}-\partial_{\nu} A_{\mu}$ is the electromagnetic field tensor. In a $3+1$-dimensional spacetime, the components of the electromagnetic field tensor $F_{\mu \nu}$ can be written as

$$
F_{\mu \nu}=\left(\begin{array}{cccc}
0 & \frac{E_{x}}{c} & \frac{E_{y}}{c} & \frac{E_{z}}{c} \\
-\frac{E_{x}}{c} & 0 & -B_{z} & B_{y} \\
-\frac{E_{y}}{c} & B_{z} & 0 & -B_{x} \\
-\frac{E_{z}}{c} & -B_{y} & B_{x} & 0
\end{array}\right) .
$$


The Euler-Lagrange equation for the vector field $A^{\mu}$ is

$$
\frac{\partial \mathscr{L}}{\partial A_{\lambda}}-\partial_{\rho}\left(\frac{\partial \mathscr{L}}{\partial\left(\partial_{\rho} A_{\lambda}\right)}\right)=0
$$

If we substitute the Lagrangian density (10) in the EulerLagrange equation (12), we will obtain the inhomogeneous Maxwell's equations as follows:

$$
\partial_{\rho} F^{\rho \lambda}=\mu_{0} J^{\lambda}
$$

The electromagnetic field tensor $F_{\mu \nu}$ satisfies the Bianchi identity

$$
\partial_{\mu} F_{\nu \lambda}+\partial_{\nu} F_{\lambda \mu}+\partial_{\lambda} F_{\mu \nu}=0
$$

Equation (14) leads to the homogeneous Maxwell's equations. Now, we obtain the Lagrangian density for electrodynamics in the presence of a minimal observable distance based on the Quesne-Tkachuk algebra. For this purpose, let us write the Lagrangian density (10) by using the representations (9), that is,

$$
\begin{gathered}
x^{\mu} \longrightarrow X^{\mu}=x^{\mu}, \\
\partial^{\mu} \longrightarrow \nabla^{\mu}:=\left(1+\beta \hbar^{2} \square\right) \partial^{\mu},
\end{gathered}
$$

where $\square:=\partial_{\mu} \partial^{\mu}$ is the d'Alembertian operator. The result reads

$$
\begin{aligned}
\mathscr{L}= & -\frac{1}{4 \mu_{0}}\left(\nabla_{\mu} A_{\nu}-\nabla_{\nu} A_{\mu}\right)\left(\nabla^{\mu} A^{\nu}-\nabla^{\nu} A^{\mu}\right)-J^{\mu} A_{\mu} \\
= & -\frac{1}{4 \mu_{0}}\left[\left(1+\beta \hbar^{2} \square\right) \partial_{\mu} A_{v}-\left(1+\beta \hbar^{2} \square\right) \partial_{\nu} A_{\mu}\right] \\
& \times\left[\left(1+\beta \hbar^{2} \square\right) \partial^{\mu} A^{\nu}-\left(1+\beta \hbar^{2} \square\right) \partial^{v} A^{\mu}\right]-J^{\mu} A_{\mu} \\
= & -\frac{1}{4 \mu_{0}} F_{\mu \nu} F^{\mu \nu}-\frac{1}{4 \mu_{0}}(\hbar \sqrt{2 \beta})^{2} F_{\mu \nu} \square F^{\mu \nu} \\
& -J^{\mu} A_{\mu}+\mathcal{O}\left((\hbar \sqrt{2 \beta})^{4}\right) .
\end{aligned}
$$

The term $-\left(1 / 4 \mu_{0}\right)(\hbar \sqrt{2 \beta})^{2} F_{\mu \nu} \square F^{\mu \nu}$ in the above Lagrangian can be considered as a minimal length effect.

If we neglect terms of order $(\hbar \sqrt{2 \beta})^{4}$ and higher in (16), we will obtain the following Lagrangian density:

$$
\mathscr{L}=-\frac{1}{4 \mu_{0}} F_{\mu \nu} F^{\mu \nu}-\frac{1}{4 \mu_{0}}(\hbar \sqrt{2 \beta})^{2} F_{\mu \nu} \square F^{\mu \nu}-J^{\mu} A_{\mu} .
$$

The Lagrangian density (17) is similar to the Abelian LeeWick model which was introduced by Lee and Wick as a finite theory of quantum electrodynamics [25-29]. Equation (17) can be written as follows:

$$
\begin{aligned}
\mathscr{L}= & -\frac{1}{4 \mu_{0}} F_{\mu \nu} F^{\mu \nu}+\frac{1}{4 \mu_{0}}(\hbar \sqrt{2 \beta})^{2}\left(\partial_{\alpha} F_{\mu \nu}\right)\left(\partial^{\alpha} F^{\mu \nu}\right) \\
& +\partial_{\alpha} \chi^{\alpha}-J^{\mu} A_{\mu},
\end{aligned}
$$

where

$$
\chi^{\alpha}:=-\frac{1}{4 \mu_{0}}(\hbar \sqrt{2 \beta})^{2} F_{\mu \nu} \partial^{\alpha} F^{\mu \nu} .
$$

After dropping the total derivative term $\partial_{\alpha} \chi^{\alpha}$, the Lagrangian density (18) will be equivalent to the following Lagrangian density:

$$
\mathscr{L}=-\frac{1}{4 \mu_{0}} F_{\mu \nu} F^{\mu \nu}+\frac{1}{4 \mu_{0}}(\hbar \sqrt{2 \beta})^{2}\left(\partial_{\alpha} F_{\mu \nu}\right)\left(\partial^{\alpha} F^{\mu \nu}\right)-J^{\mu} A_{\mu} .
$$

Using the Bianchi identity (14) and dropping the total derivative terms, the expression (20) can also be written as follows:

$$
\mathscr{L}=-\frac{1}{4 \mu_{0}} F_{\mu \nu} F^{\mu \nu}+\frac{1}{2 \mu_{0}} a^{2}\left(\partial_{\sigma} F^{\rho \sigma}\right)\left(\partial^{\beta} F_{\rho \beta}\right)-J^{\mu} A_{\mu},
$$

where $a:=\hbar \sqrt{2 \beta}$. Equation (21) is the Lagrangian density originally introduced by Podolsky [30-33] and $a$ is called Podolsky's characteristic length [34-38]. The Euler-Lagrange equation for the Lagrangian density $(20)$ is $[39,40]$

$$
\frac{\partial \mathscr{L}}{\partial A_{\lambda}}-\partial_{\rho}\left(\frac{\partial \mathscr{L}}{\partial\left(\partial_{\rho} A_{\lambda}\right)}\right)+\partial_{\sigma} \partial_{\rho}\left(\frac{\partial \mathscr{L}}{\partial\left(\partial_{\sigma} \partial_{\rho} A_{\lambda}\right)}\right)=0 .
$$

If we substitute (20) into (22), we will obtain the inhomogeneous Maxwell's equations in the presence of a minimal observable distance as follows:

$$
\partial_{\rho} F^{\rho \lambda}+(\hbar \sqrt{2 \beta})^{2} \square \partial_{\rho} F^{\rho \lambda}=\mu_{0} J^{\lambda} .
$$

It should be mentioned that (23) have been previously obtained from a different perspective by Kober [41]. Equations (14) and (23) can be written in the vector form as follows:

$$
\begin{gathered}
\nabla \cdot \mathbf{E}+(\hbar \sqrt{2 \beta})^{2} \square(\nabla \cdot \mathbf{E})=\frac{\rho}{\varepsilon_{0}}, \\
\nabla \times \mathbf{E}=-\frac{\partial \mathbf{B}}{\partial t}, \\
\nabla \times \mathbf{B}+(\hbar \sqrt{2 \beta})^{2} \square\left(\nabla \times \mathbf{B}-\frac{1}{c^{2}} \frac{\partial \mathbf{E}}{\partial t}\right)=\mu_{0} \mathbf{J}+\frac{1}{c^{2}} \frac{\partial \mathbf{E}}{\partial t}, \\
\nabla \cdot \mathbf{B}=0 .
\end{gathered}
$$

The generalized Maxwell's equations (24)-(27) have been introduced earlier by Tkachuk in [23] with a different approach. In the limit $\hbar \sqrt{2 \beta} \rightarrow 0$, the generalized inhomogeneous Maxwell's equations (24) and (26) become the usual inhomogeneous Maxwell's equations.

\section{Free Space Solutions of the Generalized Inhomogeneous Maxwell's Equations}

In this section, we obtain the plane wave solutions of the generalized inhomogeneous Maxwell's equations (23) in a 3+1-dimensional spacetime. 
In free space $(\rho=0, \mathbf{J}=0),(23)$ can be written as

$$
\partial_{\rho} F^{\rho \lambda}+(\hbar \sqrt{2 \beta})^{2} \square \partial_{\rho} F^{\rho \lambda}=0
$$

In the Lorentz gauge $\left(\partial_{\rho} A^{\rho}=0\right)$, the field equations (28) become

$$
\square A^{\lambda}+(\hbar \sqrt{2 \beta})^{2} \square \square A^{\lambda}=0 .
$$

Now, we try to find a plane wave solution of (29) as follows:

$$
A^{\lambda}(x)=A e^{-(i / \hbar) p \cdot x} \epsilon^{\lambda}(p),
$$

where $\epsilon^{\lambda}(p)$ is the polarization four-vector and $A$ is a normalization constant. In the above equation $p^{\mu}=(E / c, \mathbf{p})$ is the momentum four-vector. If we substitute (30) in (29), we will obtain

$$
p^{2}\left(1-\frac{a^{2}}{\hbar^{2}} p^{2}\right)=0
$$

where $p^{2}=p_{\mu} p^{\mu}=(E / c)^{2}-\mathbf{p}^{2}$

Equation (31) leads to the following energy-momentum relations:

$$
\begin{gathered}
E^{2}=c^{2} \mathbf{p}^{2}, \\
E^{2}=m_{\mathrm{eff}}^{2} c^{4}+c^{2} \mathbf{p}^{2},
\end{gathered}
$$

where

$$
m_{\mathrm{eff}}:=\frac{\hbar}{a c}
$$

Equation (32) describes a massless vector particle whereas (33) describes a massive vector particle with the effective mass $m_{\text {eff }}$.

\section{Upper Bound Estimation of the Minimal Length in Generalized Electrodynamics}

Substituting $\beta^{\prime}=2 \beta$ into (6) and remembering $a=\hbar \sqrt{2 \beta}$, we have

$$
\left(\Delta X^{i}\right)_{0}=\sqrt{\left(\frac{D+2}{2}\right) a^{2}\left[1+\mathcal{O}\left(a^{2}\right)\right]}, \quad \forall i \in\{1,2, \ldots, D\} .
$$

If we neglect terms of order $a^{4}$ and higher in (35), the isotropic minimal length in a $3+1$-dimensional spacetime becomes

$$
\left(\Delta X^{i}\right)_{0} \simeq \frac{\sqrt{10}}{2} a, \quad \forall i \in\{1,2,3\}
$$

Now we are ready to estimate the upper bounds on the isotropic minimal length in generalized electrodynamics.
5.1. Upper Bound on the Isotropic Minimal Length Based on the Anomalous Magnetic Moment of the Electron. In a series of papers, Accioly and coworkers [27, 29, 34] have estimated an upper bound on Podolsky's characteristic length $a$ by computing the anomalous magnetic moment of the electron in the framework of Podolsky's electrodynamics. This upper bound on $a$ is $[27,29,34]$

$$
a \leq 4.7 \times 10^{-18} \mathrm{~m}
$$

Inserting (37) into (34) and (36), we find

$$
\begin{gathered}
m_{\mathrm{eff}} \geq 41.8 \frac{\mathrm{GeV}}{c^{2}}, \\
\left(\Delta X^{i}\right)_{0} \leq 7.4 \times 10^{-18} \mathrm{~m} .
\end{gathered}
$$

5.2. Upper Bound on the Isotropic Minimal Length Based on the Ground State Energy of the Hydrogen Atom. In [37], Cuzinatto and coworkers have studied the influence of Podolsky's electrostatic potential on the ground state energy of the hydrogen atom. In their study, the upper limit on $a$ is

$$
a \leq 5.56 \times 10^{-15} \mathrm{~m} \text {. }
$$

Inserting (40) into (34) and (36), we find

$$
\begin{gathered}
m_{\mathrm{eff}} \geq 35.51 \frac{\mathrm{MeV}}{c^{2}}, \\
\left(\Delta X^{i}\right)_{0} \leq 8.79 \times 10^{-15} \mathrm{~m} .
\end{gathered}
$$

It should be noted that the upper bound (42) is about three orders of magnitude larger than the upper bound (39) that is,

$$
\begin{aligned}
& \left(\Delta X^{i}\right)_{0 \text { Ground State Energy of the Hydrogen Atom }} \\
& \sim 10^{3}\left(\Delta X^{i}\right)_{0} \text { Anomalous Magnetic Moment of the Electron' }
\end{aligned}
$$

while the lower bound (41) is about three orders of magnitude smaller than the lower bound (38) that is,

$$
\begin{aligned}
& m_{\mathrm{eff}-\text { Ground State Energy of the Hydrogen Atom }} \\
& \quad \sim 10^{-3} m_{\mathrm{eff}-\text { Anomalous Magnetic Moment of the Electron }}
\end{aligned}
$$

\section{Relationship between Nonlocal Electrodynamics and Electrodynamics in the Presence of a Minimal Length}

In a series of papers, Smailagic and Spallucci [42-44] have introduced an approach to formulate noncommutative quantum field theory. Using the Smailagic-Spallucci approach, Gaete and Spallucci introduced a nonlocal Lagrangian density for the vector field $A^{\mu}$ with an external source $J^{\mu}$ as follows:

$$
\mathscr{L}=-\frac{1}{4 \mu_{0}} F_{\mu \nu} \exp (\theta \square) F^{\mu \nu}-J^{\mu} A_{\mu}
$$


where $\theta$ is a constant parameter with dimensions of (length) $)^{2}$ [45]. We assume that the function $\exp (\theta \square)$ in (45) can be expanded in a power series as follows:

$$
\exp (\theta \square)=\sum_{l=0}^{\infty} \frac{\theta^{l}}{l !} \square^{l}
$$

where $\square^{l}$ denotes the $\square$ operator applied $l$ times [46].

If we insert (46) into (45), we will obtain the following Lagrangian density:

$$
\begin{aligned}
\mathscr{L}= & -\frac{1}{4 \mu_{0}} F_{\mu \nu} F^{\mu \nu}-\frac{1}{4 \mu_{0}} \theta F_{\mu \nu} \square F^{\mu \nu} \\
& -\frac{1}{4 \mu_{0}} \sum_{l=2}^{\infty} \frac{\theta^{l}}{l !} F_{\mu \nu} \square^{l} F^{\mu \nu}-J^{\mu} A_{\mu} .
\end{aligned}
$$

After neglecting terms of order $\theta^{2}$ and higher in (47), we obtain

$$
\mathscr{L}=-\frac{1}{4 \mu_{0}} F_{\mu \nu} F^{\mu \nu}-\frac{1}{4 \mu_{0}} \theta F_{\mu \nu} \square F^{\mu \nu}-J^{\mu} A_{\mu} .
$$

A comparison between (17) and (48) shows that there is an equivalence between the Gaete-Spallucci electrodynamics to the first order in $\theta$ and the Lee-Wick electrodynamics (or electrodynamics in the presence of a minimal length). The relationship between the noncommutative parameter $\theta$ in (48) and $a=\hbar \sqrt{2 \beta}$ in (17) is

$$
a=\sqrt{\theta} .
$$

Inserting equation (49) into (34) and (36), we find

$$
\begin{gathered}
m_{\mathrm{eff}}=\frac{\hbar}{\sqrt{\theta} c}, \\
\left(\Delta X^{i}\right)_{0} \simeq \frac{\sqrt{10 \theta}}{2}, \quad \forall i \in\{1,2,3\} .
\end{gathered}
$$

Using (40) in (49), we obtain the following upper bound for the noncommutative parameter $\theta$ :

$$
\theta_{\text {Ground State Energy of the Hydrogen Atom }} \leq 3.09 \times 10^{-29} \mathrm{~m}^{2} \text {. }
$$

The above upper bound on the noncommutative parameter $\theta$, that is, $3.09 \times 10^{-29} \mathrm{~m}^{2}$, is near to the neutron-proton scattering cross section $\left(10^{-25} \mathrm{~cm}^{2}\right)$ [47]. It is necessary to note that the electrodynamics in the presence of a minimal observable distance is only correct to the first order in the deformation parameter $\beta$, while the Gaete-Spallucci electrodynamics is valid to all orders in the noncommutative parameter $\theta$.

\section{Conclusions}

Heisenberg believed that every theory of elementary particles should contain a minimal observable distance of the magnitude $\ell_{0} \sim 10^{-13} \mathrm{~cm}[47-50]$. He hoped that the introduction of a minimal length would eliminate divergences that appear in quantum electrodynamics. Today we know that every theory of quantum gravity predicts the existence of a minimal measurable length which leads to a GUP. An immediate consequence of the GUP is a generalization of position and derivative operators according to (15) for $\beta^{\prime}=2 \beta$. It was shown that the Lagrangian formulation of electrodynamics with an external source in the presence of a minimal measurable length leads to the inhomogeneous fourth-order field equations. We demonstrated the similarity between electrodynamics in the presence of a minimal length and Lee-Wick electrodynamics. We have shown that the free space solutions of the inhomogeneous Maxwell's equations in the presence of a minimal length describe two particles, a massless vector particle and a massive vector particle with the effective mass $m_{\text {eff }}=\hbar / a c$. Now, let us compare the upper bounds on the isotropic minimal length in this paper with the results of [47-51]. The upper limit on the isotropic minimal length in (39) is near to the electroweak length scale $\left(\ell_{\text {electroweak }} \sim 10^{-18} \mathrm{~m}\right)$ [51], while the upper limit $(42)$ is near to the minimal observable distance which was proposed by Heisenberg $\left(\ell_{0} \sim 10^{-13} \mathrm{~cm}\right)$ [47-50]. It is interesting to note that the lower bound on the effective mass $m_{\text {eff }}$ in (38), that is, $41.8\left(\mathrm{GeV} / c^{2}\right)$, is of the same order of magnitude as the mass of the $W^{ \pm}$and $Z^{0}$ vector bosons $\left(M_{W}=\right.$ $\left.80.425 \pm 0.038\left(\mathrm{GeV} / c^{2}\right), M_{z}=91.1876 \pm 0.0021\left(\mathrm{GeV} / c^{2}\right)\right)$ [52]. Finally, we have investigated the relationship between the Gaete-Spallucci nonlocal electrodynamics and electrodynamics with a minimal length.

Note Added. After this work was completed, we became aware of an interesting article by Maziashvili and Megrelidze [53], in which the authors study the electromagnetic field in the presence of a momentum cutoff. For their discussion, they use the following modified Heisenberg algebra:

$$
\begin{gathered}
{\left[X^{i}, P^{j}\right]=i \hbar\left(\frac{2 \beta \mathbf{P}^{2}}{\sqrt{1+4 \beta \mathbf{P}^{2}}-1} \delta^{i j}+2 \beta P^{i} P^{j}\right),} \\
{\left[X^{i}, X^{j}\right]=0,} \\
{\left[P^{i}, P^{j}\right]=0,}
\end{gathered}
$$

where $i, j=1,2,3$ and $\beta$ is a deformation parameter [54]. In our work we have formulated electrodynamics in the framework of Quesne-Tkachuk algebra which is a Lorentzcovariant deformed algebra whereas the authors of [53] have studied electrodynamics in the framework of (52) algebra which is not a Lorentz-covariant algebra.

\section{Acknowledgment}

The authors would like to thank the referees for their useful comments.

\section{References}

[1] M. Sprenger, P. Nicolini, and M. Bleicher, "Physics on the smallest scales: an introduction to minimal length phenomenology," European Journal of Physics, vol. 33, no. 4, article 853, 2012. 
[2] S. Hossenfelder, "Minimal length scale scenarios for quantum gravity," http://128.84.158.119/abs/1203.6191.

[3] C. Castro, "On modified Weyl-Heisenberg algebras, noncommutativity, matrix-valued Planck constant and QM in Clifford spaces," Journal of Physics A, vol. 39, no. 45, pp. 14205-14229, 2006.

[4] Y. Ko, S. Lee, and S. Nam, "Modified black hole thermodynamics with generalized uncertainty principle," International Journal of Theoretical Physics, vol. 49, no. 6, pp. 1384-1395, 2010.

[5] S. Hossenfelder, "Running coupling with minimal length," Physical Review D, vol. 70, no. 10, Article ID 105003, 11 pages, 2004.

[6] M. S. Berger and M. Maziashvili, "Free particle wave function in light of the minimum-length deformed quantum mechanics and some of its phenomenological implications," Physical Review D, vol. 84, no. 4, Article ID 044043, 8 pages, 2011.

[7] M. Kober, "Electroweak theory with a minimal length," International Journal of Modern Physics A, vol. 26, no. 24, article 4251, 2011.

[8] S. Das and E. C. Vagenas, "Universality of quantum gravity corrections," Physical Review Letters, vol. 101, no. 22, Article ID 221301, 4 pages, 2008.

[9] A. F. Ali, S. Das, and E. C. Vagenas, "Discreteness of space from the generalized uncertainty principle," Physics Letters. B, vol. 678, no. 5, pp. 497-499, 2009.

[10] S. Das, E. C. Vagenas, and A. F. Ali, "Discreteness of space from GUP II: relativistic wave equations," Physics Letters B, vol. 690, no. 4, pp. 407-412, 2010.

[11] A. F. Ali, S. Das, and E. C. Vagenas, "Proposal for testing quantum gravity in the lab," Physical Review D, vol. 84, no. 4, Article ID 044013, 10 pages, 2011.

[12] C. Quesne and V. M. Tkachuk, "Composite system in deformed space with minimal length," Physical Review A, vol. 81, no. 1, Article ID 012106, 8 pages, 2010.

[13] S. K. Moayedi, M. R. Setare, and H. Moayeri, "Quantum gravitational corrections to the real klein-gordon field in the presence of a minimal length," International Journal of Theoretical Physics, vol. 49, no. 9, pp. 2080-2088, 2010.

[14] S. K. Moayedi, M. R. Setare, and H. Moayeri, "Formulation of an electrostatic field with a charge density in the presence of a minimal length based on the Kempf algebra," Europhysics Letters, vol. 98, no. 5, article 50001, 2012.

[15] S. Hossenfelder, M. Bleicher, S. Hofmann, J. Ruppert, S. Scherer, and H. Stöcker, "Signatures in the Planck regime," Physics Letters $B$, vol. 575, no. 1-2, pp. 85-99, 2003.

[16] M. R. Setare, "Corrections to the Cardy-Verlinde formula from the generalized uncertainty principle," Physical Review D, vol. 70, no. 8, Article ID 087501, p. 4, 2004.

[17] S. Basilakos, S. Das, and E. C. Vagenas, "Quantum gravity corrections and entropy at the Planck time," Journal of Cosmology and Astroparticle Physics, vol. 2010, no. 09, article 027, 2010.

[18] W. Chemissany, S. Das, A. F. Ali, and E. C. Vagenas, "Effect of the generalized uncertainty principle on post-inflation preheating," Journal of Cosmology and Astroparticle Physics, vol. 2011, no. 12, article 017, 2011.

[19] H. S. Snyder, "The electromagnetic field in quantized spacetime," Physical Review, vol. 72, pp. 68-71, 1947.

[20] C. Quesne and V. M. Tkachuk, "Lorentz-covariant deformed algebra with minimal length and application to the $(1+1)$ dimensional Dirac oscillator," Journal of Physics A, vol. 39, no. 34, pp. 10909-10922, 2006.
[21] C. Quesne and V. M. Tkachuk, "Lorentz-covariant deformed algebra with minimal length," Czechoslovak Journal of Physics, vol. 56, no. 10-11, pp. 1269-1274, 2006.

[22] H. S. Snyder, "Quantized space-time," Physical Review, vol. 71, pp. 38-41, 1947.

[23] V. M. Tkachuk, "A field equation in a deformed space with minimal length," Journal of Physical Studies, vol. 11, no. 1, pp. 41-44, 2007.

[24] D. Griffiths, Introduction to Elementary Particles, John Wiley \& Sons, New York, NY, USA, 1987.

[25] T. Lee and G. Wick, "Negative metric and the unitarity of the S-matrix," Nuclear Physics B, vol. 9, pp. 209-243, 1969.

[26] T. D. Lee and G. C. Wick, "Finite theory of quantum electrodynamics," Physical Review D, vol. 2, pp. 1033-1048, 1970.

[27] A. Accioly and E. Scatena, "Limits on the coupling constant of higher-derivative electromagnetism," Modern Physics Letters A, vol. 25, no. 04, article 269, 2010.

[28] A. Accioly, P. Gaete, J. Helayel-Neto, E. Scatena, and R. Turcati, "Exploring Lee-Wick finite electrodynamics," http://arxiv.org/abs/1012.1045v2.

[29] A. Accioly, P. Gaete, J. Helayel-Neto, E. Scatena, and R. Turcati, "Investigations in the Lee-Wick electrodynamics," Modern Physics Letters A, vol. 26, article 1985, 2011.

[30] B. Podolsky, "A generalized electrodynamics. I. Nonquantum," vol. 62, pp. 68-71, 1942.

[31] B. Podolsky and C. Kikuchi, "A generalized electrodynamics. II. Quantum," vol. 65, pp. 228-235, 1944.

[32] B. Podolsky and C. Kikuchi, "Auxiliary conditions and electrostatic interaction in generalized quantum electrodynamics," Physical Review, vol. 67, pp. 184-192, 1945.

[33] B. Podolsky and P. Schwed, "Review of a generalized electrodynamics," Reviews of Modern Physics, vol. 20, pp. 40-50, 1948.

[34] A. Accioly and H. Mukai, "Podolsky's higher-order electromagnetism from first principles," Il Nuovo Cimento della Società Italiana di Fisica B, vol. 112, no. 7, pp. 1061-1068, 1997.

[35] A. Accioly and H. Mukai, "One and the same route: two outstanding electrodynamics," Brazilian Journal of Physics, vol. 28, no. 1, pp. 35-43, 1998.

[36] R. R. Cuzinatto, C. A. M. de Melo, and P. J. Pompeia, "Second order gauge theory," Annals of Physics, vol. 322, no. 5, pp. 12111232, 2007.

[37] R. R. Cuzinatto, C. A. M. de Melo, L. G. Medeiros, and P. J. Pompeia, "How can one probe podolsky electrodynamics?" International Journal of Modern Physics A, vol. 26, no. 21, article 3641, 2011.

[38] M. V. S. Fonseca and A. V. Paredes, "Is it possible to accommodate massive photons in the framework of a gauge-invariant electrodynamics?" Brazilian Journal of Physics, vol. 40, no. 3, article 319, 2010.

[39] J. Magueijo, "Could quantum gravity phenomenology be tested with high intensity lasers?” Physical Review D, vol. 73, no. 12, Article ID 124020, 11 pages, 2006.

[40] C. M. Reyes, "Testing symmetries in effective models of higher derivative field theories," Physical Review D, vol. 80, no. 10, Article ID 105008, 13 pages, 2009.

[41] M. Kober, "Gauge theories under incorporation of a generalized uncertainty principle," Physical Review D, vol. 82, no. 8, Article ID 085017, 12 pages, 2010.

[42] A. Smailagic and E. Spallucci, "UV divergence-free QFT on noncommutative plane," Journal of Physics A, vol. 36, no. 39, article L517, 2003, 10.1088/0305-4470/36/39/103. 
[43] A. Smailagic and E. Spallucci, "Feynman path integral on the non-commutative plane," Journal of Physics A, vol. 36, no. 33, pp. L467-L471, 2003.

[44] A. Smailagic and E. Spallucci, "Lorentz invariance, unitarity and UV-finiteness of QFT on noncommutative spacetime," Journal of Physics A, vol. 37, no. 28, pp. 7169-7178, 2004.

[45] P. Gaete and E. Spallucci, "Finite axionic electrodynamics from a new non-commutative approach," Journal of Physics A, vol. 45, no. 6, p. 065401, 14, 2012.

[46] J. E. Lidsey, "Non-local inflation around a local maximum," International Journal of Modern Physics D, vol. 17, article 577, 2008.

[47] T. G. Pavlopoulos, "Breakdown of Lorentz invariance," Physical Review, vol. 159, no. 5, pp. 1106-1110, 1967.

[48] W. Heisenberg, "Zur Quantentheorie der Elementarteilchen," Zeitschrift fur Naturforschung, vol. 5, pp. 251-259, 1950.

[49] W. Heisenberg, "Über die in der theorie der elementarteilchen auftretende universelle Länge," Annalen Der Physik, vol. 32, pp. 20-33, 1938.

[50] T. G. Pavlopoulos, "Are we observing Lorentz violation in gamma ray bursts? ” Physics Letters B, vol. 625, pp. 13-18, 2005.

[51] B. Zwiebach, A First Course in String Theory, Cambridge University Press, Cambridge, UK, 2nd edition, 2009.

[52] W. N. Cottingham and D. A. Greenwood, An Introduction to the Standard Model of Particle Physics, Cambridge University Press, Cambridge, UK, 2nd edition, 2007.

[53] M. Maziashvili and L. Megrelidze, "A few comments about the Planck-length deformed quantization," http://arxiv.org/abs/ $1212.0958 \mathrm{v} 1$.

[54] A. Kempf and G. Mangano, "Minimal length uncertainty relation and ultraviolet regularization," Physical Review D, vol. 55, no. 12, pp. 7909-7920, 1997. 

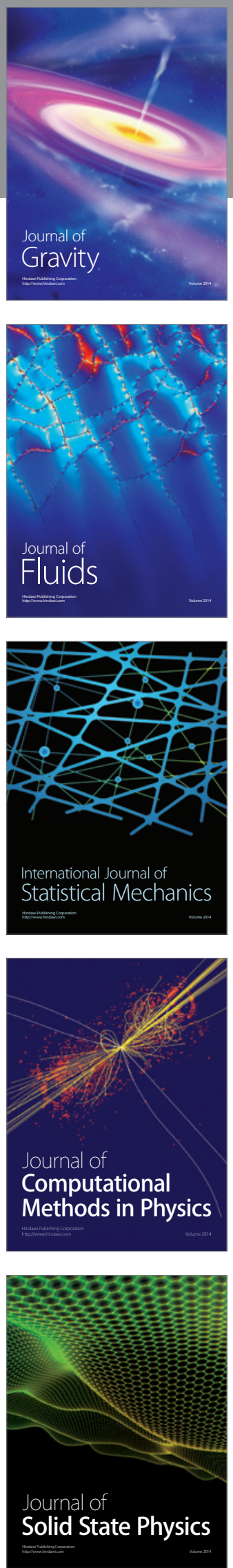

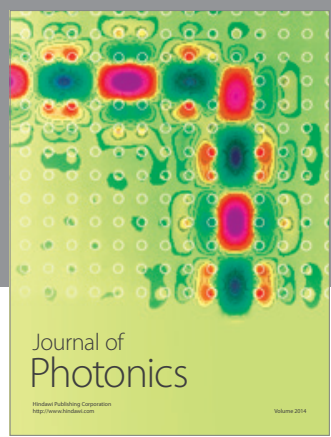

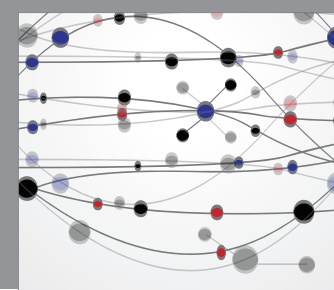

The Scientific World Journal

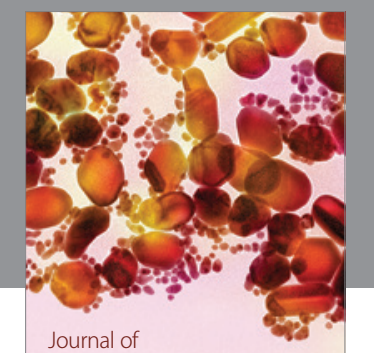

Soft Matter
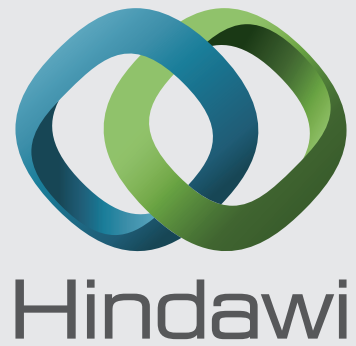

Submit your manuscripts at

http://www.hindawi.com
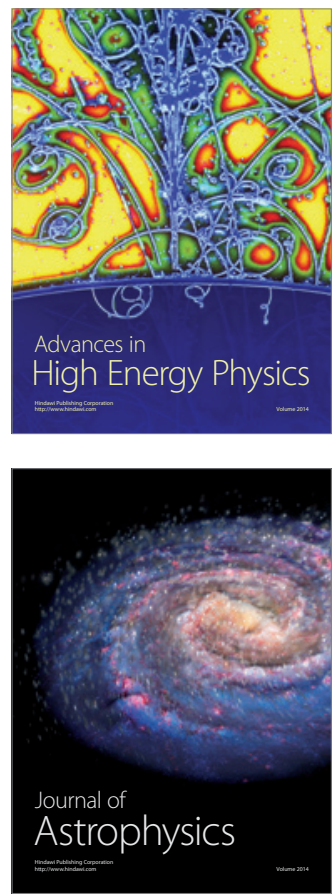
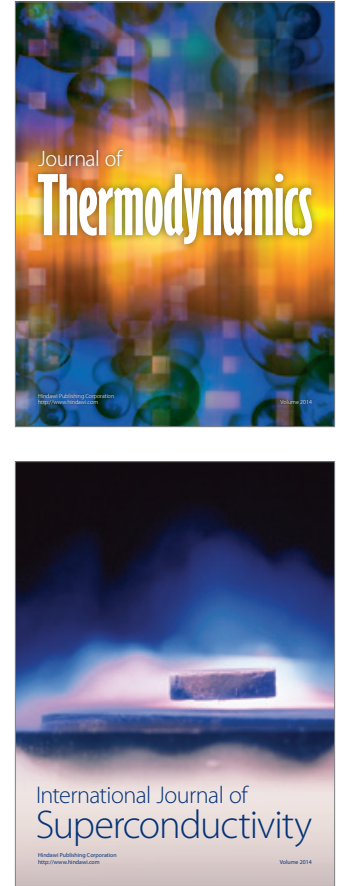
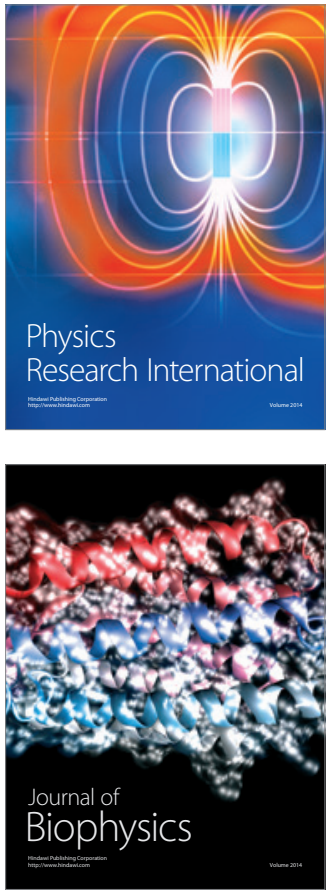
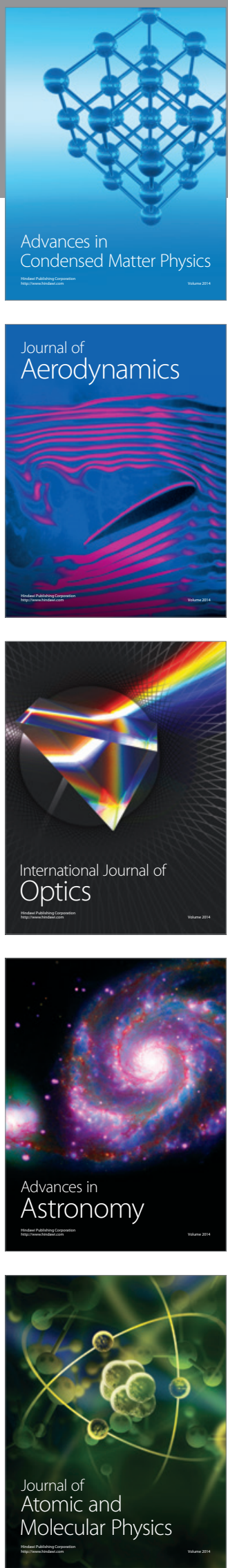